\title{
Emotion regulation and mental well-being before and six months after bariatric surgery
}

\author{
Christiane Efferdinger $^{1}$ - Dorothea König ${ }^{1}$ Alexander Klaus ${ }^{2} \cdot$ Reinhold Jagsch $^{1}(\mathbb{D}$
}

Received: 6 October 2016 / Accepted: 1 March 2017 / Published online: 7 April 2017

(C) The Author(s) 2017. This article is an open access publication

\begin{abstract}
Purpose According to the current state of research, mental health improves due to bariatric surgery. However, improvements in weight and psychosocial aspects often show a gradual decline with time. As emotion regulation (ER) appears to be a key variable in the successful outcome of weight loss treatments, the present study aimed at investigating ER-strategies applied by bariatric surgery candidates pre- and post-surgery and examining interactions between ER, depressive symptoms, health-related quality of life (HrQoL), and post-surgical weight loss.

Methods Prior to and 6 months after bariatric surgery, 45 patients (76\% women) completed self-report questionnaires assessing depressive symptoms (Beck Depression Inventory-II), HrQoL (Short Form-36 Health Survey), and ER-strategies (Emotion Regulation Inventory for Negative Emotions).

Results Six months post-surgery, the patients reported significant improvements in depressive symptomatology, HrQoL, and satisfaction with ER compared to pre-surgery. Groups differing in their course of ER-satisfaction also differed in psychosocial dimensions pre- to post-surgery, increased satisfaction being related to less impairment and enhanced communication of negative emotions as a form of an adaptive regulation. Patients with higher weight loss applied the strategy of controlled expression more
\end{abstract}

Reinhold Jagsch

reinhold.jagsch@univie.ac.at

1 Department of Applied Psychology: Health, Development, Enhancement, Intervention, Faculty of Psychology, University of Vienna, Liebiggasse 5, 1010 Vienna, Austria

2 Krankenhaus der Barmherzigen Schwestern, Stumpergasse 13, 1060 Vienna, Austria frequently post-surgery than pre-surgery and compared to patients with lower weight loss.

Conclusions Postoperative weight loss leads to improvements in ER-satisfaction and mental well-being. As satisfaction with ER seems to be associated with less impaired mental well-being among bariatric surgery candidates, presumably even more positive psychosocial outcomes could be obtained post-surgery by implementing trainings explicitly encouraging the use of adaptive ER-strategies.

Keywords Bariatric surgery · Emotion regulation · Mental well-being $\cdot$ Depressive symptoms $\cdot$ Health-related quality of life

\section{Introduction}

Morbid obesity is increasing faster than any other disease worldwide. It is associated with an elevated risk of morbidity and mortality as well as impaired mental well-being [1]. Candidates for bariatric surgery are particularly afflicted, with research indicating that up to two-thirds of the patients suffer from one or more Axis I disorders [2, 3]. Affective disorders are the most commonly reported disorders, with a lifetime prevalence of 55\% [4] - a percentage that rivals the prevalence of most of the major medical comorbidities among bariatric patients. Patients requiring bariatric surgery also consistently report a poor health-related quality of life (HrQoL), the physical components, measured with the Short Form-36 Health Survey (SF-36), being more impaired than the mental components [5, 6].

To date, bariatric surgery is the only proven treatment that achieves sustained weight loss in the majority of morbidly obese patients [7]. Meta-analyses provide evidence for the positive outcomes of bariatric surgery on 
numerous medical conditions [8,9] and aspects of mental health [10]. Mack et al. [11] and Aasprang et al. [12] found significant improvements of depressive symptoms and $\mathrm{HrQoL}$ as they examined patients up to 5 years after surgery. Peak improvements are usually reported 1 year after surgery, corresponding to the initial course of weight loss. However, as patients begin to regain weight over time, improvements in mental well-being gradually decline as well [13-15]. Nevertheless, weight, depressive symptoms, and the physical dimension of HrQoL still remain substantially improved in the long term, whereas the mental dimension of HrQoL appears to decline with time [13], sometimes even reaching baseline again [16]. Some researchers, however, propose that psychosocial factors do not worsen because of weight regain but, vice versa, impairments in mental well-being contribute to an unsuccessful weight loss or weight regain at some point post-surgery [17, 18]. Either way, emotion regulation (ER) seems to be a crucial factor in the successful outcome of weight loss treatments [19].

Deficits in ER play an important role in psychopathology $[20,21]$ such as affective disorders [22] and eating disorders [23-25]. Binge eating disorder, highly prevalent among obese individuals with a rate twice to five times as high as in the population with BMI $<30 \mathrm{~kg} / \mathrm{m}^{2}$ [26], was found to be related to maladaptive ER-strategies such as suppression of emotions [24, 25]. Nonacceptance of emotional responses emerged as a strong predictor of general eating pathology in obese individuals with binge eating disorder [27] and was recently found to play a role in non-normative eating behaviors such as emotional eating in a preoperative sample [28]. Baldofski et al. [28] state that obese individuals are likely to experience strong negative emotions due to the internalization of weight-based stereotypes while at the same time they seem to lack adaptive ER-strategies, hence engaging in emotional eating and binge eating as a way of dealing with emotions.

Given the high prevalence of affective disorders $[4,10$, 29] as well as non-normative eating behaviors and binge eating (disorder) among obese individuals $[26,27]$ and in particular among bariatric surgery candidates [10, 30-33], each of which is known to be associated with difficulties in ER, further research in this respect is required. With the help of new findings, psychological interventions focusing on ER could be implemented and specifically adapted to meet the needs of obese individuals [34, 35], supporting patients both before and after bariatric surgery.

The main focus of the present study therefore lays on determining ER-strategies applied by bariatric surgery candidates pre- and post-surgery and providing information on interactions between ER, depressive symptoms, HrQoL, and post-surgical weight loss.

\section{Methods}

\section{Participants}

Candidates for bariatric surgery were patients with a BMI $\geq 40$ or $\geq 35 \mathrm{~kg} / \mathrm{m}^{2}$ with severe obesity-associated medical conditions. Each surgical candidate underwent a multidisciplinary evaluation. Patients approved for surgery had no medical and psychological contraindications for bariatric surgery. Subjects were excluded from the study if they had previously had bariatric surgery.

\section{Measures}

The Beck Depression Inventory-II (BDI-II) [36] is a 21-item survey designed to assess the severity of depressive symptomatology during the last two weeks. Sum scores range between 0 and 63 . The severity ranges are as follows: 0-13, minimal depression; 14-19, mild depression; 20-28, moderate depression; and 29-63, severe depression [36]. Internal consistency (Cronbach's alpha) in the present sample was excellent $(\alpha=0.91)$.

To assess HrQoL, the German version of the Short Form-36 Health Survey (SF-36) was used [37]. The SF-36 is a generic measure consisting of the following eight subscales (scores: 0-100): physical functioning, role-physical, bodily pain, general health, vitality, social functioning, role-emotional, and mental health. The physical component summary score (PCS) was computed as the mean of the first four subscales $(\alpha=0.85)$, and the mental component summary score (MCS) as the mean of the four latter subscales $(\alpha=0.84)$.

ER-strategies were assessed using the Emotion Regulation Inventory for Negative Emotions (ERI-NE) [38]. This 22-item questionnaire is composed of the following five scales (scores: 0-100): distraction (distracting oneself), reappraisal (seeing the situation/emotion in a more positive light), empathic suppression (suppressing emotions so as not to impose on others), uncontrolled expression (expressing emotions unrestrainedly), and controlled expression (communicating emotions). In this study, internal consistency for the scales was good to excellent $(0.81 \leq \alpha \leq 0.97)$. An additional item refers to the satisfaction with the own regulation of negative emotions ( $1=$ very unsatisfied to $7=$ very satisfied $)$.

Higher scores in the measures indicate a more pronounced depressive symptomatology, better HrQoL, more frequent use of ER-strategies, and higher ER-satisfaction, respectively. 


\section{Procedure}

A pre-test/post-test design was used. All subjects were given questionnaires assessing depressive symptoms, HrQoL, and ER-strategies the day before surgery. Six months post-surgery, the questionnaires were mailed to the patients again, including a covering letter and a selfaddressed stamped envelope.

\section{Statistical analysis}

Data were analyzed using SPSS 22. Total percent weight loss was calculated as (weight loss $(\mathrm{kg}) /$ weight pre-surgery $(\mathrm{kg}) \times 100)$ and percent excess weight loss $(\mathrm{EWL})$ as (weight loss $(\mathrm{kg}) /$ excess weight $(\mathrm{kg}) \times 100)$. Statistical significance was defined as $p<0.05$. To compare changes in the psychosocial constructs pre- to post-surgery, dependentsamples $t$ tests were performed. To evaluate the effect of differences between two groups, Cohen's $d$ was calculated, with values $\geq 0.20$ indicating a small, $\geq 0.50$ a medium, and $\geq 0.80$ a large effect. General linear models (GLM) for repeated measures were applied in order to establish whether groups differing in their course of ER-satisfaction also differed in the psychosocial constructs over time. Post hoc tests (Scheffé) were calculated for subscales revealing a significant group main effect. GLM for repeated measures were also conducted to analyze possible differences in the psychosocial constructs over time depending on the amount of weight loss (two groups according to EWL $\geq 50 \%$ or $<50 \%)$. To evaluate the effect of differences identified by means of GLM, partial eta ${ }^{2}\left(\eta_{\mathrm{p}}{ }^{2}\right)$ was calculated, with values $\geq 0.01$ indicating a small, $\geq 0.06$ a medium, and $\geq 0.14$ a large effect. For controlling the alpha error caused by multiple testing, the Bonferroni-Holm method was used.

\section{Results}

Sixty-six patients completed the questionnaires pre-surgery and 45 of them (68\%) also completed the questionnaires post-surgery. Statistical comparisons of preoperative data (demographics, BMI, level of depression, HrQoL, and ER-strategies used) between the subset of patients who did not respond at post-surgery and those who responded demonstrated no significant differences. The responder sample $(n=45)$ had a mean age of 44.07 years $( \pm 13.28$, range 20-68 years) and comprised 34 (76\%) females; 96\% identified themselves as Austrian. The mean BMI presurgery was $45.59 \mathrm{~kg} / \mathrm{m}^{2}( \pm 7.45$, BMI ranging from 35.35 to $65.86 \mathrm{~kg} / \mathrm{m}^{2}$ ). Roux-en-Y gastric bypass was performed in 33 cases $(73 \%)$, and sleeve resection was carried out in 12 cases (27\%). Six months post-surgery, patients' mean BMI was $33.65 \mathrm{~kg} / \mathrm{m}^{2}( \pm 6.98)$, ranging from 23.83 to $56.61 \mathrm{~kg} / \mathrm{m}^{2}$, indicating a significant BMI decrease pre- to post-surgery $[t(43)=24.604, p<0.001, d=1.63]$. On average, patients lost $26 \%( \pm 7$, range $13-40 \%)$ of their initial weight; mean EWL was $62 \%$ ( \pm 19 , range $21-109 \%)$.

Regarding depressive symptomatology and HrQoL, we found significant improvements pre- to post-surgery with large effects (see Table 1). While patients on average reported a mild depression pre-surgery (BDI-II: $17.00 \pm 12.05)$, the mean postoperative BDI-II score $(6.53 \pm 7.96)$ corresponded to a minimal depressive symptomatology. In terms of HrQoL, the MCS was found to be significantly lower than the PCS $[t(41)=2.739, p=0.009$, $d=0.30]$ within the postoperative sample, while no significant differences were found within the preoperative sample $[t(41)=-1.039, p=0.305]$.

Regarding the use of ER-strategies, no statistically significant improvements were found pre- to post-surgery. However, 6 months post-surgery the patients were

Table 1 Descriptive statistics $(M \pm \mathrm{SD})$ for psychosocial constructs and test statistics for group comparisons pre- versus post-surgery (dependent-samples $t$ tests)

\begin{tabular}{|c|c|c|c|c|c|c|c|c|}
\hline Measures & Subscales & Pre-surgery $(n=45)$ & Post-surgery $(n=45)$ & $t$ & df & $p$ & $p_{\text {adj }}$ & $d$ \\
\hline BDI-II & & $17.00 \pm 12.05$ & $6.53 \pm 7.96$ & 6.362 & 44 & $<0.001$ & 0.0063 & 0.99 \\
\hline \multirow[t]{2}{*}{ SF-36 } & Physical component summary score & $54.75 \pm 25.77$ & $84.58 \pm 14.27$ & -7.309 & 38 & $<0.001$ & 0.0056 & 1.39 \\
\hline & Mental component summary score & $57.74 \pm 25.24$ & $80.17 \pm 16.85$ & -5.791 & 41 & $<0.001$ & 0.0071 & 1.03 \\
\hline \multirow[t]{6}{*}{ ERI-NE } & Distraction & $57.92 \pm 19.83$ & $61.25 \pm 22.64$ & -0.939 & 44 & 0.353 & 0.0167 & 0.16 \\
\hline & Reappraisal & $56.84 \pm 20.97$ & $59.31 \pm 23.48$ & -0.747 & 44 & 0.459 & 0.0250 & 0.11 \\
\hline & Empathic suppression & $57.36 \pm 19.41$ & $51.25 \pm 27.00$ & 1.393 & 44 & 0.170 & 0.0125 & 0.26 \\
\hline & Uncontrolled expression & $26.67 \pm 16.55$ & $28.00 \pm 20.46$ & -0.502 & 44 & 0.618 & 0.0500 & 0.07 \\
\hline & Controlled expression & $50.54 \pm 27.16$ & $59.89 \pm 33.12$ & -1.796 & 44 & 0.079 & 0.0100 & 0.31 \\
\hline & Satisfaction with emotion regulation & $4.60 \pm 1.71$ & $5.64 \pm 1.15$ & -4.175 & 44 & $<0.001$ & 0.0083 & 0.70 \\
\hline
\end{tabular}

BDI-II Beck Depression Inventory-II, SF-36 Short Form-36 Health Survey (varying $d f$ due to missing data), ERI-NE Emotion Regulation Inventory for Negative Emotions, $p_{\text {adj }}$ Bonferroni-Holm adjusted $p$ scores, $d$ effect size Cohen's $d$ 
significantly more satisfied with their ER than pre-surgery, with $d=0.70$ indicating a medium effect.

Examining the significant improvement in ER-satisfaction more closely, three groups of patients could be identified: the positive change group $(n=14)$ was unsatisfied pre-surgery, but satisfied post-surgery; about half of the sample $(n=23)$ was satisfied both pre- and post-surgery (stable positive); the smallest group $(n=8)$ showed no positive change with regard to their ER-satisfaction over time, hence remaining unsatisfied.

To compare these three groups in regard to psychosocial constructs pre- to post-surgery, $2 \times 3$ GLM were applied (see Table 2). In terms of depression, a significant time main effect $(p<0.001)$ as well as a significant group main effect $(p=0.002)$ could be observed. Post hoc tests revealed differences between the groups stable positive and no positive change $(p=0.003)$. Figure 1 indicates a considerable decrease in the depression score over time, especially within the positive change group $(p=0.002)$; however, the interaction of time and group did not reach statistical significance $(p=0.086)$. For both, the PCS and the MCS, significant time main effects were found $(p s<0.001)$. Additionally, the stable positive group showed a significantly higher MCS than the no positive change group $(p=0.001)$.

Varying results were obtained regarding ER-strategies. Significant effects were found for neither distraction and empathic suppression nor uncontrolled expression. In case of reappraisal, the group main effect was significant $(p=0.005)$, displaying high scores for the stable positive group which significantly differed from the other two groups according to post hoc tests. Regarding controlled expression, the significant group main effect $(p=0.002)$ and applied post hoc tests indicated that individuals without positive change of ER-satisfaction communicated negative emotions less frequently than the other two groups. Pre- to post-surgery, the use of controlled expression significantly increased in the positive change group only $(p=0.013)$. The interaction of time and group, however, was non-significant $(p=0.060)$ (Fig. 2).

In order to compare psychosocial changes over time depending on the amount of weight loss, the sample (one missing value) was divided into patients with an EWL $\geq 50 \%(n=29,66 \%)$ and patients with an EWL $<50 \%$ $(n=15,34 \%)$. To establish whether patients with a higher weight loss also showed greater psychosocial improvements over time, the two EWL groups were compared using GLM for repeated measures. Patients' scores of depressive symptomatology and of all $\mathrm{HrQoL}$ subscales significantly improved over time (all $p s<0.001$ ) with huge effects $\left(\eta_{\mathrm{p}}{ }^{2}\right)$ ranging from 0.26 to 0.75 . Results, however, showed no significant differences between the two EWL groups in terms of depressive symptomatology and HrQoL, indicating that patients with less weight loss had equally benefited from bariatric surgery as patients with a higher EWL. A significant interaction effect was found for the scale controlled expression $(F(1,42)=4.268, p=0.045$, $\eta_{\mathrm{p}}{ }^{2}=0.09$ indicating a medium effect). Patients with an EWL $\geq 50 \%$ made more use of the strategy post-surgery $(68.45 \pm 30.48)$ compared to pre-surgery $(51.38 \pm 26.66)$, while the opposite trend was found for patients with an EWL $<50 \%(42.33 \pm 33.05$ post-surgery, $47.63 \pm 29.33$ presurgery). Post-surgery, patients with EWL $\geq 50 \%$ applied the strategy more frequently than patients with EWL $<50 \%$ $[t(42)=2.619, p=0.012, d=0.83$ indicating a large effect $]$.

\section{Discussion}

Patients reported severe impairments in depressive symptomatology and HrQoL pre-surgery. Six months after bariatric surgery, their mental well-being had considerably improved. Individuals reported significantly fewer depressive symptoms and improvements in HrQoL; however, the MCS of the SF-36 was found to be lower than the PCS post-surgery. While studies indicate a deterioration of the MCS with time after an initial improvement $[13,16]$, the present study suggests that patients already find it harder to recover from the psychosocial than physical consequences of obesity in the first place. Yet, a considerable improvement was shown in both the physical and the psychosocial dimensions 6 months after surgery.

Patients reported a considerable improvement of satisfaction with ER over time. However, with regard to the course of ER-satisfaction over time, three different groups (positive change, stable positive, and no positive change) could be identified. Group comparisons in regard to psychosocial constructs over time suggest that higher ERsatisfaction (stable or positively changing) is associated with more favorable psychosocial outcomes 6 months post-surgery (e.g., less depressive symptomatology and increased use of controlled expression to regulate negative emotions in an adaptive way). Enhancing ER-satisfaction might therefore be a promising approach in future weight loss treatments-training patients in ER, thereby encouraging their confidence in being able to successfully control emotions and apply adaptive ER-strategies effectively [39], might contribute to improving ER-satisfaction.

Findings suggest that individuals with a higher EWL develop a more adaptive way of dealing with negative feelings post-surgery. Patients with EWL $\geq 50 \%$ increasingly regulated their emotions by talking about them-an effect that could not be found for patients with EWL $<50 \%$. Presumably, individuals with a higher weight loss feel less ashamed and shy in social situations, finding it easier to share and communicate their feelings. 


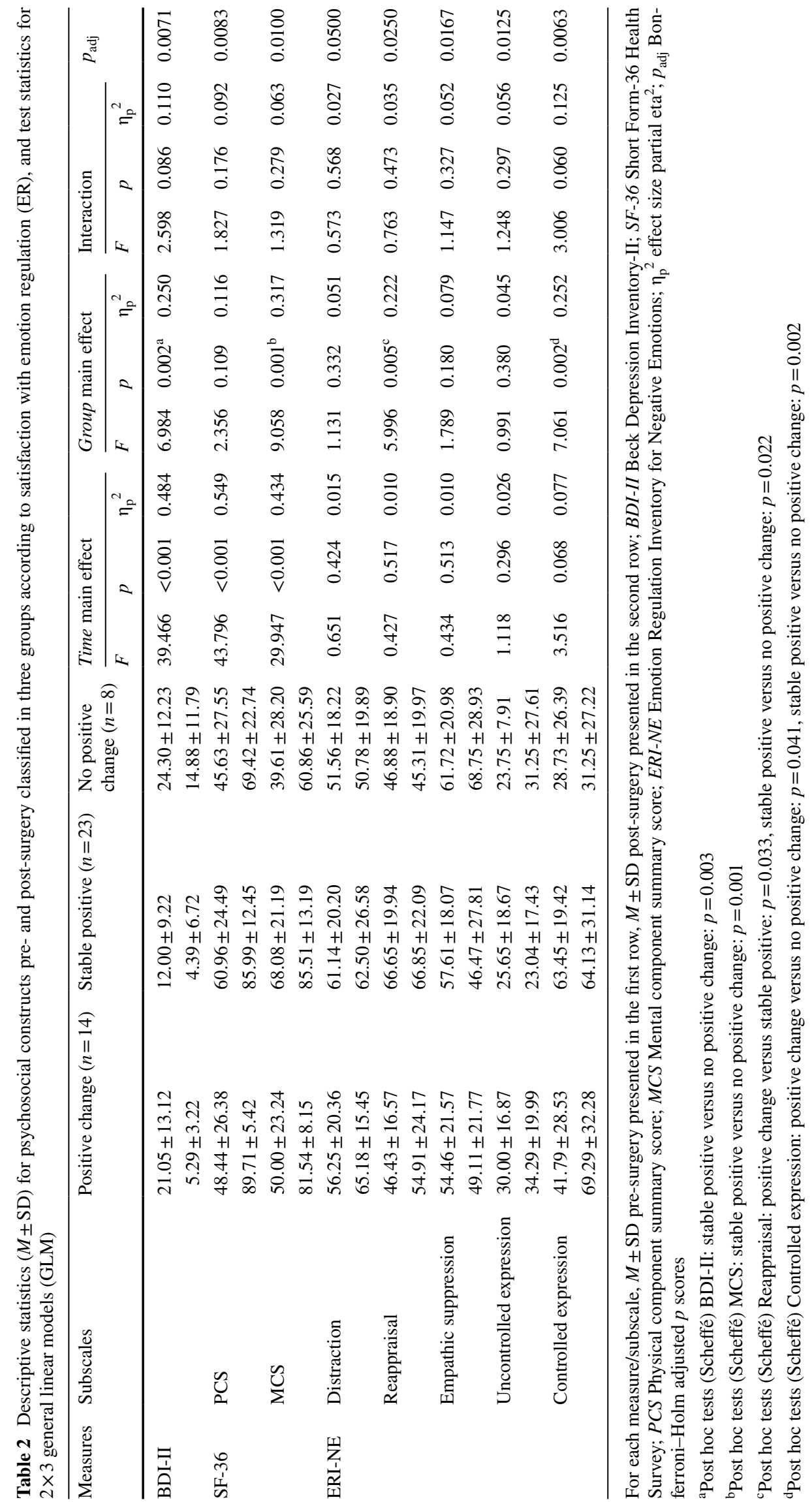




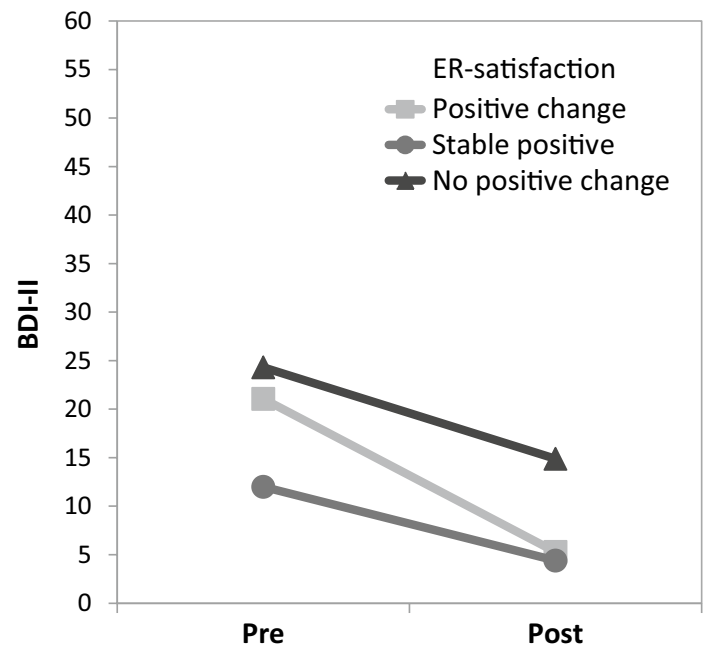

Fig. 1 Beck Depression Inventory-II (BDI-II) scores pre- and postsurgery for three groups according to satisfaction with emotion regulation (ER). Time main effect: $p<0.001$; group main effect: $p=0.002$, post hoc tests (Scheffé): no positive change versus stable positive $(p=0.003)$

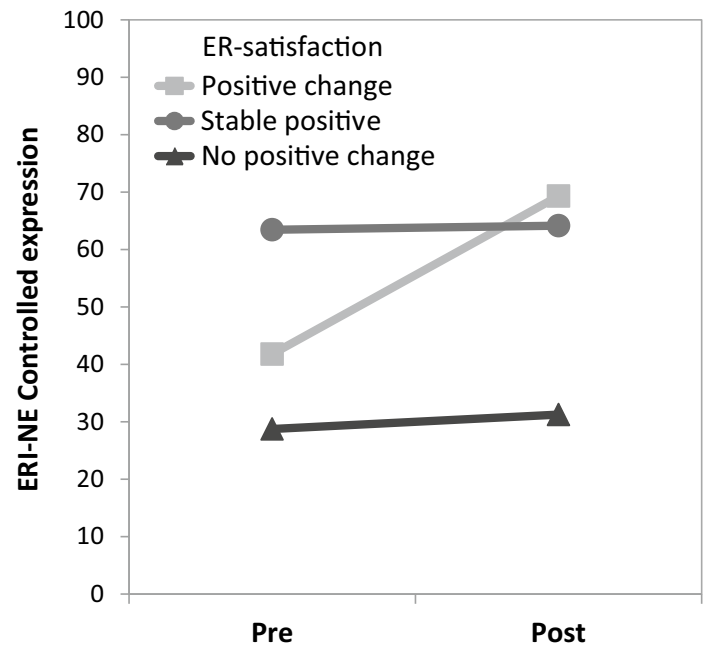

Fig. 2 Controlled expression scores pre- and post-surgery for three groups according to satisfaction with emotion regulation (ER). ERI$N E$ Emotion Regulation Inventory for Negative Emotions. Group main effect: $p=0.002$, post hoc tests (Scheffé): no positive change versus stable positive $(p=0.002)$ and positive change $(p=0.041)$

While these results indicate that massive weight loss due to bariatric surgery itself already leads to improvements in ER-satisfaction and hence mental well-being, it seems very likely that even better results could be obtained by implementing additional ER-trainings. Recent pilot studies provide evidence that psychological interventions with an ER-focus in the course of bariatric services achieve beneficial outcomes such as a decrease in depressive symptoms and eating disorders and successful weight loss $[34,35]$. A positive impact of group-based cognitive behavioral therapy (CBT) on weight loss [40] and binge eating [41] in bariatric surgery candidates has already been demonstrated. In an 8-week CBT-based group, including elements of ER, significant improvements of various psychological outcomes (e.g., frequency of anxiety and depressive symptoms, perceived difficulties in life, weight-related adjustment) have recently been recorded post-surgery [17], possibly setting the direction for further research on the topic of ER-interventions among bariatric surgery candidates.

Need for systematic interventions is high as a study by de Zwaan et al. [31] demonstrated that $25 \%$ of the participants reported "loss of control" eating on average 2 years after bariatric surgery, associated with impaired mental well-being and poorer weight loss. A preoperative binge eating disorder turned out to be a significant risk factor for the development of non-normative eating behaviors after surgery. Identifying candidates for bariatric surgery with eating disorders prior to surgery and supporting them with specific ER-trainings could contribute to both patients' satisfaction and lower costs and demands on the healthcare system.

The fact that there are no data providing information on binge eating disorder can be seen as a major limitation of the present study, considering that binge eating is known to be highly prevalent among obese individuals [26] and bariatric surgery candidates both pre- and post-surgery [30]. Binge eating disorder in turn is associated with higher rates of psychological impairment such as depression [42] and with markedly affected $\mathrm{HrQoL}$ [43], thus making its assessment and consideration even more relevant. As we were particularly interested in psychosocial variables, in the present study we mainly drew on self-report data. In addition, sample size was small and with the postoperative assessment taking place only 6 months after bariatric surgery, evaluation period was short, especially considering that "honeymoon phase" usually lasts about 2 years.

Clinical practice guidelines suggest a pre- as well as postoperative assessment by mental health professionals in order to identify patients who are likely to have difficulties adjusting to changes after bariatric surgery [44]. However, to date, postoperative psychological follow-up is not systematically provided all over Europe [45]. Screening for pathologic eating behaviors and depressive symptoms about 2 years after bariatric surgery when "honeymoon period" is over might prove especially useful. In this critical phase, weight loss has usually stabilized and patients begin to regain some weight [13], making an adaptive way of dealing with negative emotions especially relevant in order to prevent deterioration of mental well-being and significant increase of weight [46]. 
Acknowledgements The authors thank the hospital "Barmherzige Schwestern" and especially the department of clinical psychology for their assistance during recruitment and data collection.

\section{Compliance with ethical standards}

Conflict of interest The authors declare that they have no conflict of interest.

Ethical approval All procedures performed in studies involving human participants were in accordance with the ethical standards of the institutional and/or national research committee and with the 1964 Helsinki declaration and its later amendments or comparable ethical standards.

Informed consent Informed consent was obtained from all individual participants included in the study.

Open Access This article is distributed under the terms of the Creative Commons Attribution 4.0 International License (http:// creativecommons.org/licenses/by/4.0/), which permits unrestricted use, distribution, and reproduction in any medium, provided you give appropriate credit to the original author(s) and the source, provide a link to the Creative Commons license, and indicate if changes were made.

\section{References}

1. World Health Organization (2000) Obesity: preventing and managing the global epidemic. Report of a WHO consultation. WHO Technical Report Series; 894, Geneva. http://whqlibdoc.who.int/ trs/WHO_TRS_894.pdf?ua=1. Accessed 19 September 2016

2. Rosik CH (2005) Psychiatric symptoms among prospective bariatric surgery patients: rates of prevalence and their relation to social desirability, pursuit of surgery, and follow-up attendance. Obes Surg 15:677-683. doi:10.1381/0960892053923815

3. Sarwer DB, Cohn NI, Gibbons LM, Magee L, Crerand CE, Raper SE, Rosato EF, Williams NN, Wadden TA (2004) Psychiatric diagnoses and psychiatric treatment among bariatric surgery candidates. Obes Surg 14:1148-1156. doi:10.1381/0960892042386922

4. Mühlhans B, Horbach T, de Zwaan M (2009) Psychiatric disorders in bariatric surgery candidates: a review of the literature and results of a German prebariatric surgery sample. Gen Hosp Psychiatry 31:414-421. doi:10.1016/j.genhosppsych.2009.05.004

5. de Zwaan M, Lancaster KL, Mitchell JE, Howell LM, Monson N, Roerig JL, Crosby RD (2002) Health-related quality of life in morbidly obese patients: effect of gastric bypass surgery. Obes Surg 12:773-780. doi:10.1381/096089202320995547

6. Sarwer DB, Lavery M, Spitzer JC (2012) A review of the relationships between extreme obesity, quality of life, and sexual function. Obes Surg 22:668-676. doi:10.1007/ s11695-012-0588-1

7. Padwal R, Klarenbach S, Wiebe N, Birch D, Karmali S, Manns B, Hazel M, Sharma AM, Tonelli M (2011) Bariatric surgery: a systematic review and network meta-analysis of randomized trials. Obes Rev 12:602-621. doi:10.1111/j.1467-789X.2011.00866.x

8. Buchwald H, Avidor Y, Braunwald E, Jensen MD, Pories W, Fahrbach K, Schoelles K (2004) Bariatric surgery: z systematic review and meta-analysis. JAMA 292:1724-1737. doi:10.1001/ jama.292.14.1724
9. Maglione M, Sugerman HJ, Livingston EH, Nguyen NT, Li Z, Mojica WA, Hilton L, Rhodes S, Morton SC, Shekelle PG (2005) Meta-analysis: surgical treatment of obesity. Ann Intern Med 142:547-559. doi:10.7326/0003-4819-142-7-200504050-00013

10. Dawes AJ, Maggard-Gibbons M, Maher AR, Booth MJ, MiakeLye I, Beroes JM, Shekelle PG (2016) Mental health conditions among patients seeking and undergoing bariatric surgery: a metaanalysis. JAMA 315:150-163. doi:10.1001/jama.2015.18118

11. Mack I, Ölschläger S, Sauer H, von Feilitzsch M, Weimer K, Junne F, Peeraully R, Enck P, Zipfel S, Teufel M (2016) Does laparoscopic sleeve gastrectomy improve depression, stress and eating behaviour? A 4-year follow-up study. Obes Surg 26:29672973. doi:10.1007/s11695-016-2219-8

12. Aasprang A, Andersen JR, Våge V, Kolotkin RL, Natvig GK (2013) Five-year changes in health-related quality of life after biliopancreatic diversion with duodenal switch. Obes Surg 23:1662-1668. doi:10.1007/s11695-013-0994-z

13. Burgmer R, Legenbauer T, Müller A, de Zwaan M, Fischer C, Herpertz S (2014) Psychological outcome 4 years after restrictive bariatric surgery. Obes Surg 24:1670-1678. doi:10.1007/ s11695-014-1226-x

14. Karlsson J, Taft C, Rydén A, Sjöström L, Sullivan M (2007) Ten-year trends in health-related quality of life after surgical and conventional treatment for severe obesity: the SOS intervention study. Int J Obes 31:1248-1261. doi:10.1038/sj.ijo.0803573

15. Kolotkin RL, Davidson LE, Crosby RD, Hunt SC, Adams TD (2012) Six-year changes in health-related quality of life in gastric bypass patients versus obese comparison groups. Surg Obes Relat Dis 8:625-633. doi:10.1016/j.soard.2012.01.011

16. Sarwer DB, Wadden TA, Moore RH, Eisenberg MH, Raper SE, Williams NN (2010) Changes in quality of life and body image after gastric bypass surgery. Surg Obes Relat Dis 6:608-614. doi:10.1016/j.soard.2010.07.015

17. Beaulac J, Sandre D (2015) Impact of a CBT psychotherapy group on post-operative bariatric patients. SpringerPlus 4:764. doi:10.1186/s40064-015-1558-6

18. Wimmelmann CL, Dela F, Mortensen EL (2014) Psychological predictors of weight loss after bariatric surgery: a review of the recent research. Obes Res Clin Pract 8:e299-e313. doi:10.1016/j. orcp.2013.09.003

19. Silva I (2015) Importance of emotional regulation in obesity and weight loss treatment. Fractal 27:286-290. doi:10.1590/1984-0292/1503

20. Aldao A, Nolen-Hoeksama S, Schweizer S (2010) Emotion-regulation strategies across psychopathology: a meta-analytic review. Clin Psychol Rev 30:217-237. doi:10.1016/j.cpr.2009.11.004

21. Gross JJ, Jazaieri H (2014) Emotion, emotion regulation, and psychopathology: an affective science perspective. Clin Psychol Sci 2:387-401. doi:10.1177/2167702614536164

22. Joormann J, Stanton CH (2016) Examining emotion regulation in depression: a review and future directions. Behav Res Ther 86:35-49. doi:10.1016/j.brat.2016.07.007

23. Brockmeyer T, Skunde M, Wu M, Bresslein E, Rudofsky G, Herzog W, Friederich H-C (2014) Difficulties in emotion regulation across the spectrum of eating disorders. Compr Psychiatry 55:565-571. doi:10.1016/j.comppsych.2013.12.001

24. Danner UN, Sternheim L, Evers C (2014) The importance of distinguishing between the different eating disorders (sub)types when assessing emotion regulation strategies. Psychiatry Res 215:727-732. doi:10.1016/j.psychres.2014.01.005

25. Svaldi J, Griepenstroh J, Tuschen-Caffier B, Ehring T (2012) Emotion regulation deficits in eating disorders: a marker of eating pathology or general psychopathology? Psychiatry Res 197:103-111. doi:10.1016/j.psychres.2011.11.009

26. Hudson JI, Hiripi E, Pope HG, Kessler RC (2007) The prevalence and correlates of eating disorders in the National 
Comorbidity Survey Replication. Biol Psychiatry 61:348-358. doi:10.1016/j.biopsych.2006.03.040

27. Gianini LM, White MA, Masheb RM (2013) Eating, pathology, emotion regulation, and emotional overeating in obese adults with binge eating disorder. Eat Behav 14:309-313. doi:10.1016/j. eatbeh.2013.05.008

28. Baldofski S, Rudolph A, Tigges W, Herbig B, Jurowich C, Kaiser S, Dietrich A, Hilbert A (2016) Weight bias internalization, emotion dysregulation, and non-normative eating behaviors in prebariatric patients. Int J Eat Disord 49:180-185. doi:10.1002/ eat. 22484

29. Roberts RE, Kaplan GA, Shema SJ, Strawbridge WJ (2000) Are the obese at greater risk for depression? Am J Epidemiol 152:163-170. doi:10.1093/aje/152.2.163

30. de Man Lapidoth J, Ghaderi A, Norring C (2011) Binge eating in surgical weight-loss treatments. Long-term associations with weight loss, health related quality of life (HRQL), and psychopathology. Eat Weight Disord 16:e263-e269. doi:10.1007/ BF03327470

31. de Zwaan M, Hilbert A, Swan-Kremeier L, Simonich H, Lancaster K, Howell LM, Monson T, Crosby RD, Mitchell JE (2010) Comprehensive interview assessment of eating behavior 18-35 months after gastric bypass surgery for morbid obesity. Surg Obes Relat Dis 6:79-85. doi:10.1016/j.soard.2009.08.011

32. Micanti F, Iasevoli F, Cucciniello C, Costabile R, Loiarro G, Pecoraro G, Pasanisi F, Rossetti GL, Galletta D (2017) The relationship between emotional regulation and eating behaviour: a multidimensional analysis of obesity psychopathology. Eat Weight Disord. doi:10.1007/s40519-016-0275-7

33. Miller-Matero LR, Armstrong R, McCulloch K, Hyde-Nolan M, Eshelman A, Genaw J (2014) To eat or not to eat; is that really the question? An evaluation of problematic eating behaviors and mental health among bariatric surgery candidates. Eat Weight Disord 19:377-382. doi:10.1007/s40519-014-0118-3

34. Himes SM, Grothe KB, Clark MM, Swain JM, Collazo-Clavell ML, Sarr MG (2015) Stop regain: a pilot psychological intervention for bariatric patients experiencing weight regain. Obes Surg 25:922-927. doi:10.1007/s11695-015-1611-0

35. Mushquash AR, McMahan M (2015) Dialectical behavior therapy skills training reduces binge eating among patients seeking weight-management services: preliminary evidence. Eat Weight Disord 20:415-418. doi:10.1007/s40519-015-0177-0

36. Hautzinger M, Keller F, Kühner C (2006) BDI II. Beck depressions-inventar. Revision. Manual. Pearson Assessment, Frankfurt
37. Bullinger M, Kirchberger I (1998) Fragebogen zum Gesundheitszustand (SF-36). Hogrefe, Göttingen

38. König D (2011) The regulation of negative and positive emotions. Development of the Emotion Regulation Inventory and comparison of migraineurs with controls [German]. Dissertation, University of Vienna

39. Gutentag T, Halperin E, Porat R, Bigman YE, Tamir M (2016) Successful emotion regulation requires both conviction and skill: beliefs about the controllability of emotions, reappraisal, and regulation success. Cogn Emot. doi:10.1080/02699931.2016.12 13704

40. Beck NN, Johannsen M, Støving RK, Mehlsen M, Zachariae R (2012) Do postoperative psychotherapeutic interventions and support groups influence weight loss following bariatric surgery? A systematic review and meta-analysis of randomized and nonrandomized trials. Obes Surg 22:1790-1797. doi:10.1007/ s11695-012-0739-4

41. Ashton K, Drerup M, Windover A, Heinberg L (2009) Brief, four-session group CBT reduces binge eating behaviors among bariatric surgery candidates. Surg Obes Relat Dis 5:257-262. doi:10.1016/j.soard.2009.01.005

42. Grilo CM, White MA, Masheb RM (2009) DSM-IV psychiatric disorder comorbidity and its correlates in binge eating disorder. Int J Eat Disorder 42:228-234. doi:10.1002/eat.20599

43. Ágh T, Kovács G, Pawaskar M, Supina D, Inotai A, Vokó Z (2015) Epidemiology, health-related quality of life and economic burden of binge eating disorder: a systematic literature review. Eat Weight Disord 20:1-12. doi:10.1007/s40519-014-0173-9

44. Mechanick JI, Youdim A, Jones DB, Garvey WT, Hurley DL, McMahon MM, Heinberg LJ, Kushner R, Adams TD, Shikora S, Dixon JB, Brethauer S (2013) Clinical practice guidelines for the perioperative nutritional, metabolic, and nonsurgical support of the bariatric surgery patient-2013 update: cosponsored by American Association of Clinical Endocrinologists, the Obesity Society, and American Society for Metabolic \& Bariatric Surgery. Surg Obes Relat Dis 9:159-191. doi:10.1016/j. soard.2012.12.010

45. Lamore K, Kaci SS, Czernichow S, Bretault M, Bouillot JL, Naudé AJ, Gribe-Ouaknine S, Carette C, Flahault C (2017) Mental health support provided throughout the bariatric surgery clinical pathway in French specialized care centers for obesity. Obes Surg. doi:10.1007/s11695-016-2498-0

46. Saunders R (2004) "Grazing": a high-risk behavior. Obes Surg 14:98-102. doi:10.1381/096089204772787374 